\title{
Shielding vacuum fluctuations with graphene
}

\author{
Sofia Ribeiro \\ Quantum Optics and Laser Science, Blackett Laboratory, Imperial College London, \\ Prince Consort Road, London SW7 2BW, United Kingdom \\ Stefan Scheel \\ Institut für Physik, Universität Rostock, Universitätsplatz 3, D-18051 Rostock, Germany
}

(Dated: June 22, 2021)

\begin{abstract}
The Casimir-Polder interaction of ground-state and excited atoms with graphene is investigated with the aim to establish whether graphene systems can be used as a shield for vacuum fluctuations of an underlying substrate. We calculate the zero-temperature Casimir-Polder potential from the reflection coefficients of graphene within the framework of the Dirac model. For both doped and undoped graphene we show limits at which graphene could be used effectively as a shield. Additional results are given for AB-stacked bilayer graphene.

PACS numbers: 31.30.jh, 34.35.+a, 78.67.Wj, 42.50.Nn
\end{abstract}

\section{INTRODUCTION}

Graphene's extraordinary electronic and optical properties hold great promise for applications in photonics and optoelectronics. The existence of a true twodimensional (2D) material having a thickness of a single atom was believed to be impossible for a long time, because both finite temperature and quantum fluctuations would destroy the 2D structure. However, since the first groundbreaking experiments [1, the study of graphene became an active field in condensed matter. Theoretical reviews of graphene's properties can be found in Refs. [2, 3]. The technological push towards miniaturization resulted in the idea of devising small structures based on graphene. For instance, placing graphene between different substrates or by patterning a given substrate, it is possible to create artificial materials with tunable properties [4.

Hybrid quantum systems which combine cold atoms with solid structures hold great promise for the study of fundamental science, creating the possibility to built devices to measure precisely gravitational, electric and magnetic fields [5]. For instance, many of the proposed extensions to the Standard Model of particle physics include forces, due to compactified extra dimensions, that would modify Newtonian gravity on submicrometer scales [6, 7]. By performing extremely careful force measurements near surfaces, it is hoped that more stringent limits on the presence of such forces may be obtained. With this in mind, hybrid systems in which neutral atoms and graphene are held in close proximity represent an important and attractive case to study. A quick estimate shows that the Casimir-Polder force dominates gravity by several orders of magnitude at micrometer distances. It is therefore necessary to find a system that is simple enough in order to either be able to calculate its dispersion effect to high enough precision, or to provide a shield against vacuum fluctuations of another (macroscopic) body.

Graphene has been shown to be a strong absorber of electromagnetic radiation, it interacts strongly with light over a wide wavelength range, particularly in the far infrared and terahertz parts of the spectrum due to its high carrier mobility and conductivity [8]. Considering that graphene is only one atomic layer thick, its (universal) absorption coefficient of $\eta=\pi e^{2} /(\hbar c) \approx 2.3 \%$ is quite remarkable 9. In Ref. 10 new systems made of several layers of graphene are shown to be an effective shield for terahertz radiation, while letting visible light pass. These studies brought the attention to the development of transparent mid- and far-infrared photonic devices.

With graphene's absorption properties in mind we investigate the possibility of shielding electromagnetic vacuum fluctuations of a macroscopic body placed nearby. The purpose of this study is to investigate whether and under which circumstances the Casimir-Polder potential between an atom and a graphene-substrate system is dominated by the interaction with graphene such that the effect of the substrate does not play an important role. This knowledge will allow us to manipulate the Casimir-Polder potential of a layered system by placing the graphene at different graphene-substrate distances or by patterning it into different shapes.

This article is organised as follows. After briefly introducing graphene into the formalism of macroscopic QED in Sec. II. we give some numerical results for the CasimirPolder shift of an atom near a graphene sheet in Sec. III. In Sec. IV] and V], we study different the shielding of vacuum fluctuations by single-layer and bilayer graphene, respectively, and give concluding remarks in Sec. VI

\section{CASIMIR-POLDER INTERACTION WITH GRAPHENE}

It is well known that an atom placed near a macroscopic body will experience a dispersion force - the Casimir-Polder force - due to the presence of fluctuations of the electromagnetic field even at zero temperature [11. We begin by investigating the Casimir-Polder interaction of an atom next to a graphene layer at zero 
temperature. We adopt the Dirac model for graphene and calculate the Casimir-Polder interactions based on the formalism of macroscopic QED. Upon quantization of the electromagnetic field in the presence of absorbing bodies, and application of second-order perturbation theory, the Casimir-Polder potential for planar structures can be written as 12 ]

$$
\begin{gathered}
U_{\mathrm{CP}}\left(z_{A}\right)=\frac{\hbar \mu_{0}}{8 \pi^{2}} \int_{0}^{\infty} d \xi \xi^{2} \alpha_{a t}(i \xi) \\
\times \int_{0}^{\infty} d k_{\|} \frac{e^{-2 k_{\|} \gamma_{0 z} z_{A}}}{\gamma_{0 z}}\left[\mathrm{R}_{\mathrm{TE}}+\mathrm{R}_{\mathrm{TM}}\left(1-\frac{2 k_{\|}^{2} \gamma_{0 z}^{2} c^{2}}{\xi^{2}}\right)\right]
\end{gathered}
$$

where $\gamma_{i z}=\sqrt{1+\varepsilon_{i}(i \xi) \xi^{2} k_{\|}^{2} / c^{2}}$ is the $z$-component of the wavenumber in the medium with permittivity $\varepsilon_{i}$ for imaginary frequencies (the index 0 refers to the medium in which the atom is placed) and $\alpha_{a t}(\omega)$ is the isotropic atomic polarizability defined by

$$
\alpha_{a t}(\omega)=\lim _{\varepsilon \rightarrow 0} \frac{2}{\hbar} \sum_{k_{A} \neq 0_{A}} \frac{\omega_{k 0} \mathbf{d}_{0 k} \cdot \mathbf{d}_{k 0}}{\omega_{k 0}^{2}-\omega^{2}-i \omega \varepsilon} .
$$

This equation is valid for zero temperature. A replacement of the frequency integral by a Matsubara sum has to be performed for finite temperatures 12. In this case, the potential is well approximated by inserting the temperature-dependent reflection coefficients in the lowest term in the Matsubara sum $(j=0)$ while keeping the zero-temperature coefficients for all higher Matsubara terms [13. Only for $k_{B} T \gtrsim \Delta$ thermal corrections become important [14] ( $\Delta \approx 0.1 \mathrm{eV}$ is the gap parameter of quasiparticle excitations).

In order to compute the reflection coefficients $R_{T M}$ and $\mathrm{R}_{\mathrm{TE}}$ for transverse magnetic (TM) and transverse electric (TE) waves from a graphene sheet, two alternative models, the hydrodynamic model and the Dirac model, exist. The first treats graphene as an infinitesimally thin positively charged flat sheet, carrying a homogeneous fluid with some mass and negative charge densities. On the other hand, the Dirac model incorporates the conical electron dispersion relation of Dirac fermions, modelling graphene as a two-dimensional gas of massless Dirac fermions, where low-energy excitations are considered Dirac fermions that move with a Fermi velocity. The ranges of validity of these respective models is not completely resolved [5].

The reflection coefficients are calculated by matching the dyadic Green function of free space and its derivatives on either side of a two-dimensional conducting sheet [15, with the result that

$$
\begin{aligned}
\mathrm{R}_{\mathrm{TM}} & =\frac{\gamma_{0 z} \alpha_{\|}\left(k_{\|}, \omega\right)}{1+\gamma_{0 z} \alpha_{\|}\left(k_{\|}, \omega\right)} \\
\mathrm{R}_{\mathrm{TE}} & =\frac{\left(\omega / c k_{\|}\right)^{2} \alpha_{\perp}\left(k_{\|}, \omega\right)}{\gamma_{0 z}-\left(\omega / c k_{\|}\right)^{2} \alpha_{\perp}\left(k_{\|}, \omega\right)},
\end{aligned}
$$

where

$$
\alpha(\mathbf{k}, \omega)=-e^{2} \frac{\chi(\mathbf{k}, \omega)}{2 \varepsilon_{0} k_{\|}}=i \frac{\sigma(\mathbf{k}, \omega) k_{\|}}{2 \varepsilon_{0} \omega}
$$

is given by the density-density correlation function $\chi(\mathbf{k}, \omega)$ [16, 17] or, alternatively, the conductivity $\sigma(\mathbf{k}, \omega)$ [18, 19. The functions for doped and undoped graphene are derived based on the band structure of graphene. The problem with this approach is that there are no transverse functions available in the literature for single sheets, only the longitudinal functions [15]. It has been shown in Ref. 20] that, at zero as well as at finite temperature, the retardation effects in graphene systems are negligible. Hence, we neglect the retardation effects, so that the TE modes do not contribute and we can set $\gamma_{0 z} \rightarrow 1$.

The density-density correlation function for undoped graphene in the complex frequency plane $(\omega=i \xi)$ is given by [15]

$$
\chi(\mathbf{k}, i \xi)=-\frac{g}{16 \hbar} \frac{k_{\|}^{2}}{\sqrt{v_{F}^{2} k_{\|}^{2}+\xi^{2}}} .
$$

The parameter $g=4$ represents the degeneracy parameter, with a factor of 2 accounting for spin and another factor of 2 for cone degeneracy; $v_{F}$ is the Fermi velocity.

However, most materials naturally occur with charge doping where the Fermi level or chemical potential $\mu$ is away from charge neutrality $(\mu=0)$. When the graphene sheet is doped, the density-density correlation function becomes more complicated. Following Ref. [15], the density-density correlation function on the imaginary frequency axis can be written in terms of the dimensionless variables $\tilde{k}=k_{\|} / 2 k_{F}$ and $\tilde{\xi}=\hbar \xi / 2 E_{F}\left(E_{F}=\hbar v_{F} k_{F}\right.$ and $k_{F}=\sqrt{4 \pi n / g}$ ), as

$$
\chi(\mathbf{k}, \xi)=-D_{0}\left[1+\frac{\tilde{k}^{2}}{4 \sqrt{\tilde{k}^{2}+\tilde{\xi}^{2}}}(\pi-f(\tilde{k}, \tilde{\xi}))\right]
$$

where $D_{0}=\sqrt{g n /\left(\pi \hbar^{2} v_{F}^{2}\right)}$ is the density of states at the Fermi level for doping concentration $n$. The function $f(\tilde{k}, \tilde{\xi})$ is defined as

$$
\begin{gathered}
f(\tilde{k}, \tilde{\xi})=\arcsin \left(\frac{1-i \tilde{\xi}}{\tilde{k}}\right)+\arcsin \left(\frac{1+i \tilde{\xi}}{\tilde{k}}\right) \\
-\frac{i \tilde{\xi}-1}{\tilde{k}} \sqrt{1-\left(\frac{i \tilde{\xi}-1}{\tilde{k}}\right)^{2}}+\frac{i \tilde{\xi}+1}{\tilde{k}} \sqrt{1-\left(\frac{i \tilde{\xi}+1}{\tilde{k}}\right)^{2}} .
\end{gathered}
$$

In real graphene there are always deviations from the conical shapes of the band structure and other than the lowest bands also contribute, thus the functions used here are valid only for low frequencies $(\hbar \omega \lesssim 4 \mathrm{eV})$. 


\section{ATOM NEAR A SINGLE GRAPHENE SHEET}

The simplest geometry is an atom at a distance $z_{A}$ away from a perfectly flat graphene sheet. In case of undoped graphene, the relevant density-density correlation function to be used in the reflection coefficients at a graphene/vacuum interface $R_{\mathrm{TM}}$ and $R_{\mathrm{TE}}$ [Eqs. (3)], is the one shown in Eq. (5).

In the framework of this study, it is interesting to compare this result to the interaction between an atom and a perfect conductor, where $R_{\mathrm{TM}}=1$ and $R_{\mathrm{TE}}=-1$. As an example, we have chosen a ground-state rubidium atom at zero temperature. We found that the interaction between the atom and graphene is about $\sim 5 \%$ of the interaction between an atom and a perfect conductor, see Fig. 1. Using the same Dirac model for graphene, it has already been shown that, at zero temperature, the interaction between graphene and an ideal conductor is about $2.6 \%$ of the interaction between two perfect conductors separated by the same distance [21].

When the graphene sheets are doped, one has to use the reflection coefficients given in Eq. (3) with $\chi(\mathbf{k}, i \xi)$ defined by Eq. (6). The results are shown in Fig. 1 and Table I] where we present the results for doping densities $10^{10}, 10^{11}, 10^{12}$ and $10^{13} \mathrm{~cm}^{-2}$.

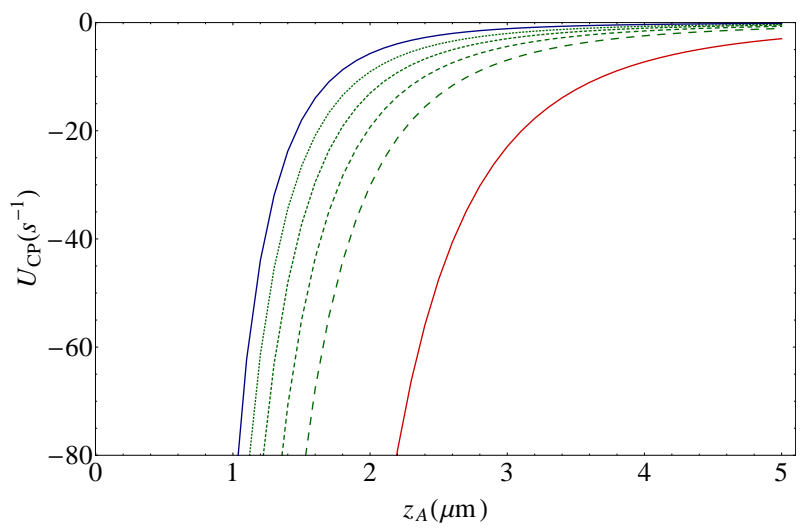

FIG. 1: (Color online) Casimir-Polder potential between a ground state rubidium atom and a doped graphene sheet. The upper solid line (blue) is the result for undoped graphene, while the dashed curves (green) are for doping densities $10^{10}$, $10^{11}, 10^{12}$ and $10^{13} \mathrm{~cm}^{-2}$, respectively, from top to bottom. The solid bottom line (red) is the result for a perfect conductor.

\section{GRAPHENE SHEET ABOVE A GOLD SUBSTRATE}

The purpose of this manuscript is to show whether one (or more) freestanding graphene sheet could be a candidate to shield the effects of a substrate below on an atom above the graphene sheets (see Fig. 2). For a single graphene layer, the system will in effect be a layered
TABLE I: Casimir-Polder potential between rubidium atoms in the ground state and graphene sheets at $z_{A}=1 \mu \mathrm{m}$.

\begin{tabular}{lr}
\hline \hline doping density $\left(\mathrm{cm}^{-2}\right)$ & $U_{\mathrm{CP}}\left(\mathrm{s}^{-1}\right)$ \\
\hline \hline no doping & -90.987 \\
$10^{10}$ & -121.940 \\
$10^{11}$ & -165.489 \\
$10^{12}$ & -244.768 \\
$10^{13}$ & -371.140 \\
\hline \hline
\end{tabular}

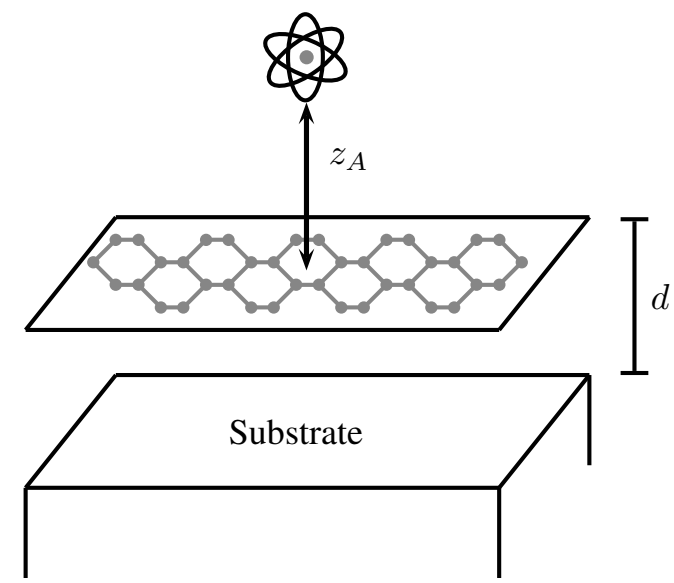

FIG. 2: Scheme of an atom standing above a free standing graphene sheet above a substrate.

medium of the structure $1 \nmid 2 \mid 3$, where the graphene sheet (denoted by $\nmid$ ) separates the free-space regions 1 and 2 , the index 3 denotes the substrate (subscript $s$ ) with permittivity $\varepsilon(\omega)$. The Fresnel coefficients for this geometry can be written as 22 .

$$
\tilde{R}=R_{G}+\frac{T_{G} R_{0 s} T_{G} e^{2 i k_{0 z} d}}{1-R_{G} R_{0 s} e^{2 i k_{0 z} d}}
$$

where $T_{G}$ is the transmission coefficient through the graphene layer. For the different modes we will have different coefficients, such that $T_{G}^{\mathrm{TE}}=1+R_{G}^{\mathrm{TE}}$ is valid only for the amplitude coefficient of the TE mode and the conditions $T_{G}^{\mathrm{TM}}=1-R_{G}^{\mathrm{TM}}$ should be used for the TM mode. The Fresnel coefficients for this structure $1 \nmid 2 \mid 3$ should be written as

$$
\begin{aligned}
\tilde{R}^{\mathrm{TE}} & =\frac{R_{G}^{\mathrm{TE}}+R_{0 s}^{\mathrm{TE}} e^{2 i k_{0 z} d}+2 R_{G}^{\mathrm{TE}} R_{0 s}^{\mathrm{TE}} e^{2 i k_{0 z} d}}{1-R_{G}^{\mathrm{TE}} R_{0 s}^{\mathrm{TE}} e^{2 i k_{0 z} d}} \\
\tilde{R}^{\mathrm{TM}} & =\frac{R_{G}^{\mathrm{TM}}+R_{0 s}^{\mathrm{TM}} e^{2 i k_{0 z} d}-2 R_{G}^{\mathrm{TM}} R_{0 s}^{\mathrm{TM}} e^{2 i k_{0 z} d}}{1-R_{G}^{\mathrm{TM}} R_{0 s}^{\mathrm{TM}} e^{2 i k_{0 z} d}} .
\end{aligned}
$$

The reflection coefficients for TE and TM waves at the interface between free space and the substrate are the 
usual Fresnel coefficients

$$
\begin{aligned}
\mathrm{R}_{\mathrm{TM}}^{0 s} & =\frac{\varepsilon_{s} \gamma_{0 z}-\gamma_{s z}}{\varepsilon_{s} \gamma_{0 z}+\gamma_{s z}}, \\
\mathrm{R}_{\mathrm{TE}}^{0 s} & =\frac{\gamma_{0 z}-\gamma_{s z}}{\gamma_{0 z}+\gamma_{s z}} .
\end{aligned}
$$

In the following, we will present our results for the Casimir-Polder interaction with both doped and undoped graphene sheets. As a substrate material we have chosen gold, a material used in several experimental setups, whose permittivity we describe by the Drude model

$$
\varepsilon(\omega)=1-\frac{\omega_{p e}^{2}}{\omega\left(\omega+i \gamma_{e}\right)}
$$

with parameters $\omega_{p e}=1.37 \times 10^{16} \mathrm{~s}^{-1}$ and $\gamma_{e}=4.12 \times$ $10^{13} \mathrm{~s}^{-1}$.

\section{A. Undoped graphene sheet}

In this simple geometry with only one graphene sheet, the total Casimir-Polder potential of the graphenesubstrate system is limited by the potential of the single graphene sheet and that for the gold substrate. At small distances $d$ between graphene and substrate the Casimir-Polder interaction felt by the atom is dominated by the interaction with the gold substrate. With increasing distance $d$, the Casimir-Polder potential is well approximated by that of a single graphene sheet.

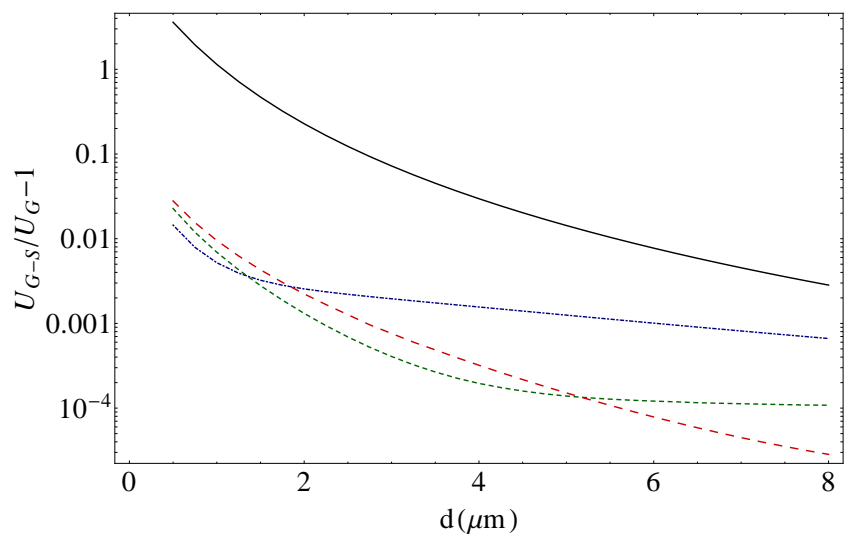

FIG. 3: (Color online) Normalized Casimir-Polder potential of a rubidium atom in the ground state (black line), 32S (blue dotted line), 43S (green dashed line) and 54S (red dashed line) at $z_{A}=1 \mu \mathrm{m}$ for different distances $d$ between the undoped graphene sheet and gold.

In order to quantify the shielding effect of graphene, we fix the atom's position at $z_{A}=1 \mu \mathrm{m}$, vary the distance $d$ between the graphene and the substrate, and normalize these results to the Casimir-Polder potential without the substrate at the same distance $z_{A}$.

Due to the recent experimental progress in working with atoms in Rydberg states, one might look at the differences that may arise from having an atom in an excited state rather than in its ground state. The atomic transition frequencies of a highly excited Rydberg atom are in a window of frequencies in which graphene absorbs well [8, 10, so that one would expect a larger CasimirPolder shift for the atom-graphene-gold system than for the corresponding atom-gold system.

For the calculation of the interaction energy between an atom in a excited state and a surface one has to add a resonant contribution to the usual non-resonant CasimirPolder potential (1)

$$
\begin{aligned}
& U_{\mathrm{CP}}^{\mathrm{R}}\left(z_{A}\right)=\frac{\mu_{0}}{4 \pi} \sum_{k \neq n} \omega_{n k}^{2} \mathbf{d}_{n k} \cdot \mathbf{d}_{k n} \int_{0}^{\infty} d \kappa_{0 z} e^{-2 \kappa_{0 z} z_{A}} \\
& \times\left[\operatorname{Re}\left[\mathrm{R}_{\mathrm{TE}}\right]+\operatorname{Re}\left[\mathrm{R}_{\mathrm{TM}}\right]\left(1+\frac{2 \kappa_{0 z}^{2} c^{2}}{\omega^{2}}\right)\right] .
\end{aligned}
$$

The Casimir-Polder potentials for a selection of Rydberg states of rubidium are shown in Fig. 3 and compared with the corresponding results for the ground state. We can clearly see that for the excited states, the shielding properties of graphene are highlighted. The differences in the potential experienced by the various states reflect the resonances of the different atomic transitions frequencies allowed for each state.

\section{B. Doped graphene sheet}

For doped graphene one has to use the density-density correlation function Eq. (6) in the reflection coefficients Eq. (3). In Fig. 4 we show the results for different doping concentrations for fixed atom-graphene and graphenegold distances. One observes that for higher doping concentration the shielding effect of graphene becomes somewhat better than at lower concentrations. This is due to the fact that the conductivity increases and therefore the graphene sheet more and more resembles a perfect conductor, see Fig. 1 .

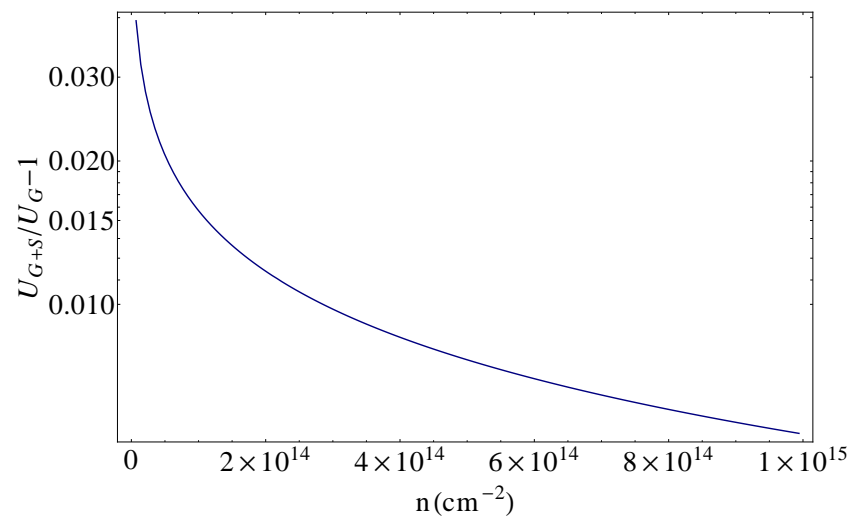

FIG. 4: (Color online) Normalized Casimir-Polder potential of a rubidium atom in the ground state at $z_{A}=1 \mu \mathrm{m}$ and $d=2 \mu \mathrm{m}$ for different doping densities. 
Note that these results change for different atomic eigenstates, the effect of which are shown in Fig. 5 for ground and excited state (the curves were calculated for a doping concentration of $n=10^{12} \mathrm{~cm}^{2}$ ). Each atom has different frequency transitions that influence the strength of the atom-graphene coupling. In the same way, different doping concentrations will also influence the absorbance of graphene, Fig. 1, so it is expected that each concentration and each atomic state to yield unique results.

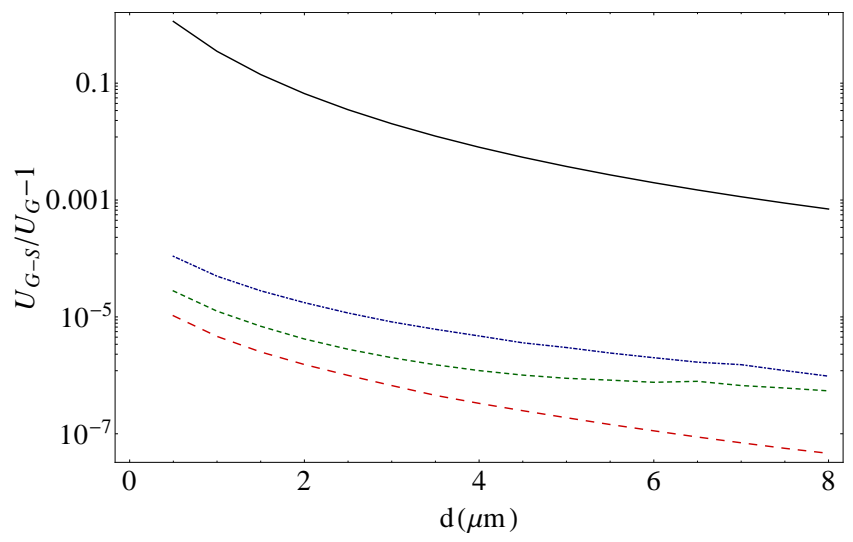

FIG. 5: (Color online) Normalized Casimir-Polder potential of a rubidium $\left({ }^{87} \mathrm{Rb}\right)$ atom in the ground state (black line), 32S (blue dotted line), 43S (green dashed line) and 54S (red dashed line) at $z_{A}=1 \mu \mathrm{m}$ for different distances $d$ between doped graphene $\left(n=10^{12} \mathrm{~cm}^{2}\right)$ and gold.

\section{BILAYER GRAPHENE ABOVE A SUBSTRATE}

During the manufacturing of graphene, layers of varying thickness are typically generated. Besides the 'pure' form of single-layer graphene, bilayers of two weakly bound sheets are common. The natural form for bilayer graphene is the AB-stacking, which is the basis of graphite. However, alternative stackings are also available where one layer is rotated by some angle relative to the other [19. Here we focus on AB-stacking in which half the atoms are aligned on top of one another whereas the other half are located above the center of the hexagonal lattice of the opposite layer.

The conductivity of AB-stacked bilayer graphene can be found in Refs. [18, 19, for both doped and undoped cases. The longitudinal conductivity for undoped ABstacking $(\mu=0)$ at zero temperature can be written as

$$
\begin{gathered}
\sigma_{x x}(\omega)=\frac{e^{2}}{2 \hbar} \\
\times\left[\left(\frac{\omega+2 \gamma}{2(\omega+\gamma)}+\frac{\omega-2 \gamma}{2(\omega-\gamma)} \Theta(\omega-2 \gamma)\right) \Theta(\omega)\right. \\
\left.+\frac{\gamma^{2}}{2 \omega^{2}}[\Theta(\omega-\gamma)+\Theta(\omega+\gamma)] \Theta(\omega-\gamma)\right] .
\end{gathered}
$$

and the perpendicular conductivity is given by

$$
\begin{gathered}
\sigma_{z z}(\omega)=\frac{e^{2}}{4 \hbar}\left(\frac{\gamma d}{\hbar v_{F}}\right)^{2} \\
\times\left[\frac{\omega}{2(\omega+\gamma)}+\frac{\omega}{2(\omega-\gamma)} \Theta(\omega-2 \gamma)\right] \Theta(\omega)
\end{gathered}
$$

where $\Theta(x)$ is the Heaviside step function, $\gamma=0.4 \mathrm{eV}$ is the interlayer hopping energy and $d=3.3 \AA$ the interlayer distance. These results for conductivity do not include the spin-orbit coupling. That effect could be also considered to calculate the conductivity in order to cover other possible effects [19].

The conductivity at imaginary frequencies as required for the nonresonant Casimir-Polder potential can be obtained from the Kramers-Kronig relation

$$
\sigma(i \xi)=\frac{2}{\pi} \int_{0}^{\infty} d \omega \frac{\omega \operatorname{Im} \sigma(\omega)}{\omega^{2}+\xi^{2}}
$$

In Fig. 6 we show the Casimir-Polder potential of an atom (either in its ground state or in a Rydberg state) next to a graphene bilayer. When compared to a single graphene sheet, a bilayer of graphene does not provide a better shielding for a ground state atom and for an excited one the results are even more unfavourable, see Fig. 3

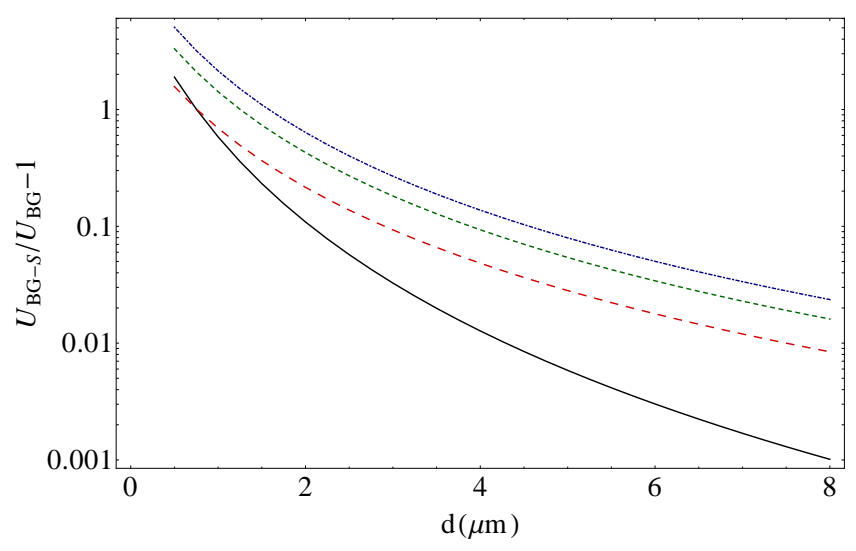

FIG. 6: (Color online) Normalized Casimir-Polder potential of a rubidium atom in the ground state (black line), 32S (blue dotted line), 43S (green dashed line) and 54S (red dashed line), at $z_{A}=1 \mu \mathrm{m}$ for different distances $d$ between the graphene bilayer and gold.

\section{CONCLUSIONS}

The knowledge of how to control and manipulate graphene systems opens the possibility of a number of novel research possibilities. A layered structure made from graphene could be used as an effective shield for the effects of a substrate beneath, by patterning the graphene into disks and ribbons, one would be able to create tunable filters, where the disks and nanoribbons would shield 
the substrate and the void regions would only feel the effects of the substrate.

We have shown that, in some situations, in a atomgraphene-substrate system the Casimir-Polder potential is dominated by the graphene potential. For graphenesubstrate distances larger than $4 \mu \mathrm{m}$ we verified that for all studied systems, one or more graphene membranes shield the effect of electromagnetic vacuum fluctuations emanating from a substrate. The optical absorption of graphene is dominated by intraband transitions in the far-infrared spectral range and by interband transitions from mid-infrared to ultraviolet [23]. The coupling of graphene with different states will lead to different couplings as we could verify from the results obtained from ground and highly excited states. Each state has different frequency transitions which will influence the strength of the atom-graphene coupling.

These results show that shielding the Casimir-Polder forces is a rather delicate issue. It has been proposed that, since hydrogen-switchable mirrors are shiny metals which become optically transparent upon exposure to hydrogen, the Casimir force between them should be stronger in air than in hydrogen. However, that was been shown in Ref. 24] not to be the case, the reason being that, although the mirrors are indeed shiny metals in air, this change in optical properties only affects the optical range of frequencies. In order to have an effective change in the Casimir interaction one would need to have the mirror which is strongly reflecting at all frequencies 25].

A similar situation is encountered with graphene. The results for patterned graphene in Ref. 8 show that it is be possible to create a system that is highly absorbing in a small frequency band, so as to tune out the resonant part of the Casimir-Polder interaction for an atom in the excited state. However, in order to be able to shield the non-resonant part one would have to have a material that is more broadband absorbing.

Several factors could still be included to make our model more realistic. Amongst them are finite temperature, corrugation of the freestanding graphene sample and the presence of impurities. However, for clean enough samples, these factors are normally considered as perturbations, not changing the essentials of the Dirac model [21].

We would like to acknowledge fruitful discussions with E.A. Hinds and S.Y. Buhmann. SR is supported by the PhD grant SFRH/BD/62377/2009 from FCT, cofinanced by FSE, POPH/QREN and EU.
[1] K. S. Novoselov, A. K. Geim, S. V. Morozov, D. Jiang, Y. Zhang, S. V. Dubonos, I. V. Grigorieva, and A. A. Firsov, Science 306, 666 (2004).

[2] A. H. C. Neto, F. Guinea, N. M. R. Peres, K. S. Novoselov, and A. K. Geim, Reviews of Modern Physics 81, 109 (2009).

[3] N. M. R. Peres, Reviews of Modern Physics 82, 2673 (2010).

[4] A. H. C. Neto and K. S. Novoselov, Reports in Progress on Physics 74, 082501 (2011).

[5] T. E. Judd, R. G. Scott, A. M. Martin, B. Kaczmarek, and T. M. Fromhold, New Journal of Physics 13, 083020 (2011).

[6] E. Fischbach and C. L. Talmadge, The search for nonnewtonian gravity (Springer, 1999), 1st ed.

[7] E. G. Adelberger, B. R. Heckel, and A. E. Nelson, Ann. Rev. Nucl. Part. Sci. 53, 77 (2003).

[8] S. Thongrattanasiri, F. H. L. Koppens, and F. J. Garcia de Abajo, Physical Review Letters 108, 047401 (2012).

[9] M. I. Katsnelson, Graphene Carbon in Two Dimensions (Cambridge University Press, 2012), 1st ed.

[10] H. Yan, X. Li, B. Chandra, G. Tulevski, Y. Wu, M. Freitag, W. Zhu, P. Avouris, and F. Xia, Nature Nanotechnology Letters 7, 330 (2012).

[11] H. B. G. Casimir and D. Polder, Physical Review 73 (1948).

[12] S. Scheel and S. Y. Buhmann, Acta Physica Slovaca 58,
675 (2008).

[13] I. V. Fialkovsky, V. N. Marachevsky, and D. V. Vassilevich, Physical Review B 84, 035446 (2011).

[14] M. Chaichian, G. L. Klimchitskaya, V. M. Mostepanenko, and A. Tureanu, Physical Review A 86, 012515 (2012).

[15] B. E. Sernelius, Physical Review B 85, 195427 (2012).

[16] B. Wunsch, T. Stauber, F. Sols, and F. Guinea, New Journal of Physics 8 (2006).

[17] E. H. Hwang and S. DasSarma, Physical Review B 75, 205418 (2007).

[18] E. J. Nicol and J. P. Carbotte, Physical Review B 77, 155409 (2008)

[19] C. J. Tabert and E. J. Nicol, Physical Review B 86, 075439 (2012).

[20] G. Gómez-Santos, Physical Review B 80, 245424 (2009).

[21] M. Bordag, I. V. Fialkovsky, D. M. Gitman, and D. V. Vassilevich, Physical Review B 80, 245406 (2009).

[22] W. C. Chew, Waves and Fields in Inhomogeneous Media (IEEE Press, 1994).

[23] K. F. Mak, L. Ju, F. Wang, and T. F. Heinz, Solid State Communications 152, 1341 (2012).

[24] D. Iannuzi, M. Lisanti, and F. Capasso, Proc. Natl. Acad. Sci. USA 101, 4019 (2004).

[25] S. de Man and D. Iannuzzi, New Journal of Physics 8 (2006). 